\title{
LEAD TIMES - THEIR BEHAVIOR AND THE IMPACT ON PLANNING AND CONTROL IN SUPPLY CHAINS
}

\author{
Peter Nielsen ${ }^{1}$, Zbigniew Michna ${ }^{2}$, Brian Bruhn Sørensen ${ }^{3}$, Ngoc Do Anh Dung ${ }^{1}$ \\ ${ }^{1}$ Department of Materials and Production, Aalborg University, Denmark \\ ${ }^{2}$ Department of Mathematics and Cybernetics, Wroclaw University of Economics, Wroclaw, Poland \\ ${ }^{3}$ SCM Consultant, Copenhagen, Denmark
}

Corresponding author:

Peter Nielsen

Aalborg University

Department of Materials and Production

Aalborg 9220, Denmark

phone: +4599408932

e-mail:peter@m-tech.aau.dk

Received: 5 December 2016 Accepted: 13 February 2017

\begin{abstract}
Lead times and their nature have received limited interest in literature despite their large impact on the performance and the management of supply chains. This paper presents a method and a case implementation of the same, to establish the behavior of real lead times in supply chains. The paper explores the behavior of lead times and illustrates how in one particular case they can and should be considered to be independent and identically distributed (i.i.d.). The conclusion is also that the stochastic nature of the lead times contributes more to lead time demand variance than demand variance.
\end{abstract}

KEYWORDS

supply chain management, stochastic lead time, simulation, inventory management.

\section{Introduction}

Supply chain management focuses on the coordination of activities across a chain of companies ordered in a number of echelons and can be addressed from a number of different perspectives [1]. This research is focused on lead time distributions and their importance for supply chain management and describing actual lead time behavior. Moreover, the focus of the research is on how knowing your lead time behavior (and identifying how it is different from your expectations) can lead to changes in the supply chain setup and lead to a changed focus in a manufacturing company's planning and control approach.

The remainder of the paper is structured as follows. First, a literature review of lead times in the context of supply chain management is presented. This includes a motivation from a mathematical perspective of why lead time distributions are critical, in some contexts more so than demand distributions. Second, a method for identifying lead time distributions and lead time behavior in supply chain is presented. Third, a case study containing both actual lead time distribution analysis and managerial insight gained from the investigation is presented. Finally, general managerial implications from stochastic lead times and conclusions are presented.

\section{Motivation and literature review}

So why are lead time distributions and their behavior so relevant for planning \& control? In both inventory and supply chain management lead time demand expectation and variance of the same are critical when determining order quantities, re-order points and managing the supply chain [2]. However, it is not always clear why both demand and lead time distributions are critical for both the behavior and performance of a supply chain. To investigate this let us define lead time demand $\left(D_{t}^{L}\right)$ as shown in (1) (see e.g. Chen et al. [3] for a similar definition):

$$
D_{t}^{L}=D_{t}+D_{t+1}+\cdots+D_{t+L-1}=\sum_{i=0}^{L_{t-1}} D_{t+i}
$$


We have to note that lead time demand is a crucial parameter when placing an order in a supply chain as it is used to determine inventory and order policies. In practice one has to investigate and address what causes the main inefficiencies of a given supply chain and in this context the behavior of lead time demand becomes critical. It is interesting to note that authors such as Mascle and Gosse [4] state that limited information about lead time demand distributions are actually available. Typically, this inefficiency of information is defined in the form of the bullwhip effect (demand variance amplification) and the increase of the inventory level variance [3]. Keeping both these parameters relatively small is one of the most significant challenges in supply chain management.

The above definition of lead time demand permits to draw some conclusions if we assume that lead times $\left(L_{t}\right)$ and demands $\left(D_{t}\right)$ are mutually independent at any given time $\mathrm{t}$ and that $D_{t}$ and $L_{t}$ are i.i.d., it as shown in (2) and (3):

$$
\begin{gathered}
E\left(D_{t}^{L}\right)=E\left(D_{t} L_{t}\right)=E\left(D_{t}\right) E\left(L_{t}\right), \\
\operatorname{Var}\left(D_{t}^{L}\right)=E\left(\operatorname{Var}\left(D_{t}^{L} \mid L_{t}\right)\right)+\operatorname{Var}\left(E\left(D_{t}^{L} \mid L_{t}\right)\right) \\
=E\left(L_{t} \operatorname{Var}\left(D_{t}\right)\right)+\operatorname{Var}\left(L_{t} E\left(D_{t}\right)\right) \\
=E L_{t} \operatorname{Var}\left(D_{t}\right)+E\left(D_{t}\right)^{2} \operatorname{Var}\left(L_{t}\right) .
\end{gathered}
$$

The estimation of the expected mean lead time demand $\left(E\left(D_{t}^{L}\right)\right)$ gives the average quantity of an order and the estimation of the variance of lead time demand $\left(\operatorname{Var}\left(D_{t}^{L}\right)\right)$ is used to determine the safety stock (see e.g. [5]). The last formulas on the lead time expectation and variance signify that through a simple sample of $D_{t}$ and $L_{t}$ (given mutual independence) it is possible to estimate both the mean and variance of lead time demand $\left(D_{t}^{L}\right)$. Moreover, it is worth noting that the variance of lead times is scaled by the squared value of the expected demand rate. An example of the relative importance of lead time variance $\left(\operatorname{Var}\left(L_{t}\right)\right)$ vs. demand variance $\left(\operatorname{Var}\left(D_{t}\right)\right)$ can be seen in Fig. 1 where lead time demand variance is calculated for an example where $E L_{t}$ and $E\left(D_{t}\right)$ are kept constant at 10 units and variance of both are varied between 0 and 400 .

The graph in Fig. 1 illustrates that lead time demand variance is very dependent on lead time variance, much more so than on demand variance. If the connections are so straight forward and lead time variance is so large part of lead time demand variance it begs the question of why there is so limited insight into how lead times actually behave? This becomes even more interesting when contrasted again the attention given to demand and lead time demand distributions in both literature and practice. The motivation for this research is twofold. First, there is clearly a need to establish a method for determining actual lead time distributions and document various occurrences of these. Second, the managerial implications of having stochastic lead times in the context of supply chain management must be determined.

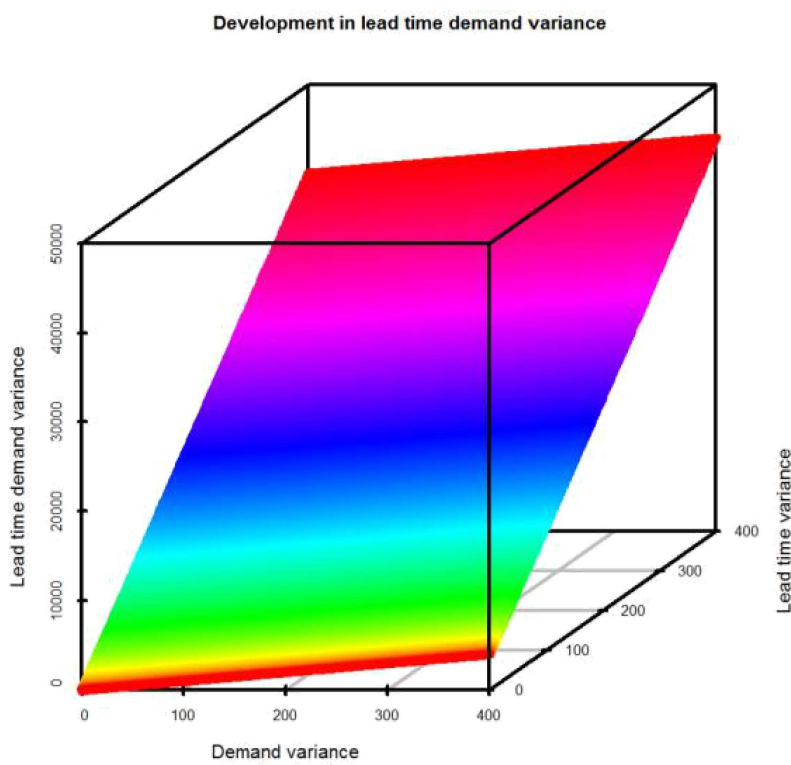

Fig. 1. Example of development in lead time demand variance.

\section{Analytical approaches to modeling supply chains with stochastic lead times}

Most research to date has considered deterministic lead time and two echelons systems. In general, the analytical models assuming deterministic lead times (e.g. [3]) have several weaknesses when put in the context of real life supply chain management. These models are typically based on the assumption of an infinite history of observations being available and assume a simpler demand structure than is realistic to experience. Moreover, lead times are treated as deterministic, while in practice lead times must behave stochastically (see e.g. [6]). A few papers have investigated stochastic lead time and multi-echelon systems. One such is Chatfield et al. [7], who note that stochastic lead times are a major source of bullwhip effect. Kim et al. [8] choose another approach by assuming that both demand and lead times are stochastic, but rather than predict demands and assume stochastic lead times they choose to predict lead time demand. Bagchi et al. [9] approximated the demand during stochastic lead time and used it to calculate the safety stock. They underline that the impact on stock out and stock out risk when the lead time variance in an independent demand system is ignored. They also show that using a normal approximation of the demand during stochastic lead time often yields 
significant errors because the true distribution is usually very much skewed to the right. Another interesting aspect addressed by Chaharsooghi and Heydari [10] determines for a specific supply chain what has the largest effect; reducing the average lead time or reducing the variance of lead time? This underlines the importance of determining actual lead time behavior to reduce the bullwhip effect in supply chains. However, no research focuses on the effect of stochastic lead time of the upstream echelon to that of the downstream one. All the identified research assumes that the lead times of echelons are independent. This is not practical because the variance of lead time of a downstream echelon can be longer due to the variance of lead time of an upstream echelon.

Wikner et al. [11] note that a twenty percent reduction in peak amplification can be achieved if lead times are reduced. Metters [12] identify the magnitude of the bullwhip effect by establishing an empirical lower bound on the profitability impact of the bullwhip effect and found that eliminating the bullwhip effect can increase product profitability by 10 $30 \%$. They also point out a lead time reduction strategy that could eliminate the bullwhip effect would also generate significant savings in inventories and other costs. Chen et al. [3] prove that increasing the lead time will increase the variability of orders from retailer to manufacturer. The general consensus in current state is that the root causes for bullwhip effect (see e.g. [13-15] are: demand forecasting, non-zero lead time, supply shortage, order batching, price fluctuation and lead time forecasting (see [16]). However, it is also obvious from such contributions as Chaharsooghi and Heydari [10] and Michna et al. [16] that stochastic lead times can be critical when evaluating the performance of a supply chain in terms of the bullwhip effect.

Song [17] study the effect of stochastic lead time in a single item inventory model and conclude that the higher variance of lead time leads to higher longrun average cost. Buchmeister et al. [18] analyze the bullwhip effect for a multi-echelon supply chain. In their study, two cases for demand fluctuation are considered: stable demand with a single $5 \%$ change and changing demand with $10 \%$ increase in first four periods and $10 \%$ decrease in four periods later. They also suggest several possible actions to reduce the bullwhip effect in supply chain. Ghaffari and Javadian [19] focus on developing new forecasting techniques to reduce bullwhip effect in a multi-echelon multi-product supply chain.

Michna et al. [16] investigate a two stage supply chain, with stochastic demands and stochastic lead times, both i.i.d. and mutually independent. They conclude that when forecasting lead times, the bullwhip effect in a two echelon supply chain depends on the mean and variance of demand and lead time and the number of observations available to forecast demands and lead times, respectively. They conclude that this holds for an arbitrary distribution of both demands and lead times. More precisely their work supports the fact that the bullwhip effect is caused by the need to forecast demands and lead times and the smoother the demand and lead time forecasts, the smaller the bullwhip effect. Moreover, only the first two moments of demand and lead time distributions are significant for the bullwhip effect. Similar conclusions regarding the importance of lead time variation and its influence on the bullwhip effect can be found in [20] and [21].

\section{Simulation approaches to modeling supply chains with stochastic lead times}

A number of researchers have used simulation to investigate the effect of stochastic lead times on supply chains. Some papers have focused on the effect of lead time on inventory. Gross and Soriano [22] analyze the effect of lead time reduction on inventory level. Eppen and Martin [23] use simulation to determine the safety stock in the case of stochastic lead time and demand. They consider two cases in which the parameters of the distributions are known and unknown. Several papers study the effect of stochastic lead time on a simple or multiple stage supply chain. Chaharsooghi and Heydari [10] use simulation and multivariate models to conclude that lead time variance is in fact a major cause of bullwhip effect. They concluded that the lead time variance has a higher impact than other factors on the supply chain performance. So and Zheng [24] analyze the supplier's lead time and forecast demand updating for a two-level supply chain model using simulation to show how stochastic lead times and the correlation of the external demands can amplify the order variance in a supply chain. Chatfield et al. [7] simulate a periodic order-up-to level inventory system to analyze the effects of stochastic lead time and information sharing in supply chain. They also take in account the quality of information. Chen et al. [3] implement simulation to quantify the bullwhip effect with the impact of forecasting, lead time and information to a simple supply chain, and then extend to multiple-stage supply chain with and without centralized customer demand information.

The overall conclusion from literature is two-fold. First, the impact of stochastic lead times on supply chains can be reduced to the following: 
- The bullwhip effect in supply chains caused by stochastic lead times only depends on the first and second moments of the lead time distribution i.e. the mean and variance.

- Lead times have in all the investigated research been assumed to be i.i.d. when treated as stochastic rather than deterministic.

Second, surprisingly limited research has been published on how lead times actually behave. While it is recognized that lead time variance is actually a major source of bullwhip effect (see e.g. [16]) the authors have not been able to identify any comprehensive research into the nature of lead times. This leads to the conclusion that lead time behavior is of outmost importance for both academia and practitioners in the field of Supply Chain Management.

\section{Method for establishing lead time be- havior}

The following paragraph contains a description of a method extending the work in Nielsen et al. [6] for establishing the behavior of lead times and gain insight into their behavior. This method is motivated by the current state of literature and a clear impact on practical planning problems of stochastic lead times. The elements that are of specific interest for us are:

(1) What is the relationship between the mean and variance of lead times and how does this vary?

(2) Can lead times be considered to be i.i.d.?

(3) What is the typical shape of a lead time distribution?

(4) How much does the variance of lead time demand change if stochastic lead times are considered?

Point 1 is interesting from the perspective that from literature it is established that when lead times are stochastic the mean and variance of the distribution are the critical parameters when determining the expected bullwhip effect of the supply chain. Likewise, from point 1 it is possible to establish whether or not lead times are in fact close enough to constant that they can be treated as such. Point 2 is significant as it is known from current state of research that when lead times are treated as stochastic, they are always assumed to be i.i.d. Point 3 is interesting from several perspectives. First, from the perspective of how extreme can lead time distributions be expected to be? Second, from the perspective of a single investigation, are the lead times across a product family behaving in a similar manner and if so can this knowledge be exploited to improve supply chain performance? Point 4 refers to Eq. (5) where it is clear that lead time demand variation depends on lead time variance. Here it is interesting to establish the increase in lead time demand variance if lead times are considered stochastic rather than deterministic. From the above it is obvious that there is a need to complete various analyses. Point 1 can be managed by monitoring the coefficient of variance of lead times $\left(\widehat{\sigma}_{t} / \widehat{L}_{t}\right.$, where $\widehat{\sigma}_{t}$ is the sample standard deviation of lead times and $\widehat{L}_{t}$ is the sample mean lead time) for various sample sizes. A value of $\widehat{\sigma}_{t} / \widehat{L}_{t}$ less than 0.05 would typically indicate that the variable can be treated as constant rather than stochastic. Point 2 is addressed by using the method proposed in Nielsen et al. [6] where independence and identical distributions are investigated separately using respectively autocorrelation (Box and Jenkins) and KolmogorovSmirnov's (KS) test for identical distributions. That the lead times are assumed to be i.i.d. also means that they are e.g. assumed to be independent of order sizes or if the particular product order is part of a bigger order of other products. These aspects are not investigated in this paper, but are obvious points for further research. Point 3 is quite simple to establish by investigating how the skewness and kurtosis of the lead time distribution behaves. Skewness is a statistical property of a distribution indicating whether the data is skewed to the left (negative values), symmetrical (zero) or skewed to the right (positive values). Skewness is relative with the third moment of the distribution (mean and variance being relative with the first and second moments respectively). Kurtosis is a measure of whether or not the data exhibits peaks. These characteristics of the lead time distributions combined with the information gained from point 1 can be used to make general inferences about the distributions' behavior. To investigate point 4 , it seems to appropriately calculate the following ratio variance arising from demand uncertainty divided by variance arising from lead time uncertainty, or more precisely on real data as shown in (4):

$$
\Delta S S=\frac{\widehat{D}_{t}^{2} \widehat{\sigma}_{L, t}^{2}}{\widehat{L}_{t} \widehat{\sigma}_{D, t}^{2}},
$$

where $\widehat{L}_{t}$ is the sample mean of the lead time for the sample, $\widehat{\sigma}_{L, t}$ is the sample estimate of the variance of lead time, $\widehat{D}_{t}$ is the sample estimate of the average demand per time unit and $\widehat{\sigma}_{D, t}$ is the sample estimate of variance of demand per time unit. We term this ratio $\Delta S S$, as it roughly can be translated into the need to increase safety stock at a given echelon to achieve the same service level under deterministic lead times. More precisely the term describes the relationship between demand and lead time uncertainty. The $\Delta S S$ term is thus a proxy for the relative 
amount of uncertainty from a demand and lead time respectively. It is obvious that this ratio is larger than 0 for non-deterministic lead times and that a value of 1 will correspond to a doubling of the lead time demand variance through the presence of stochastic lead times.

\section{Case}

To investigate the behavior of lead times we have obtained from a company the delivery lead times for 50 products over a period of 4 years and a total of 98,219 deliveries from the same factory to various customers in the same region. I.e. we assume that the delivery lead time for a given order should follow the same distribution as any other order for the same product. In lieu of supplier lead times, we assume that a similar behavior can be seen from suppliers and use this to estimate the lead time demand variance. This gives us a proxy in large scale for how we expect the next echelon in this company's supply chain will experience lead time demand variance and in turn becomes a measure on how the company inside their own supply chain is creating added variance in lead time demand. From this description alone it should be clear that while orders may arrive in a stochastic manner they are at the very least not completely randomly distributed, but somehow structured i.e. some form of dependence can exist.

\section{Coefficient of variation of lead time distributions}

In Fig. 2, four histograms corresponding to four different sample sizes from the lead time observations are shown. From the histograms several general inferences regarding the 50 products' lead time distributions can be drawn. First, all the distributions tend to have large $\widehat{\sigma}_{t} / \widehat{L}_{t}$ ratios, with the majority of distributions in the median having a coefficient of variance of 1.18, 1.27, 1.34 and 1.41 for the sample sizes 25, 50, 100 and 200 respectively. This of course indicates that the underlying lead time distributions are rather volatile as e.g. the $\widehat{\sigma}_{t} / \widehat{L}_{t}$ ratio is 1 for an exponential distribution. Second, it would be a gross misrepresentation of reality to assume that lead times are constant for any of the investigated products.

\section{Test of i.i.d.}

Having established that lead times in the investigated case are strictly non-deterministic, the next step is establishing whether or not lead times can be assumed to be i.i.d. using the method proposed in Nielsen et al. [6].
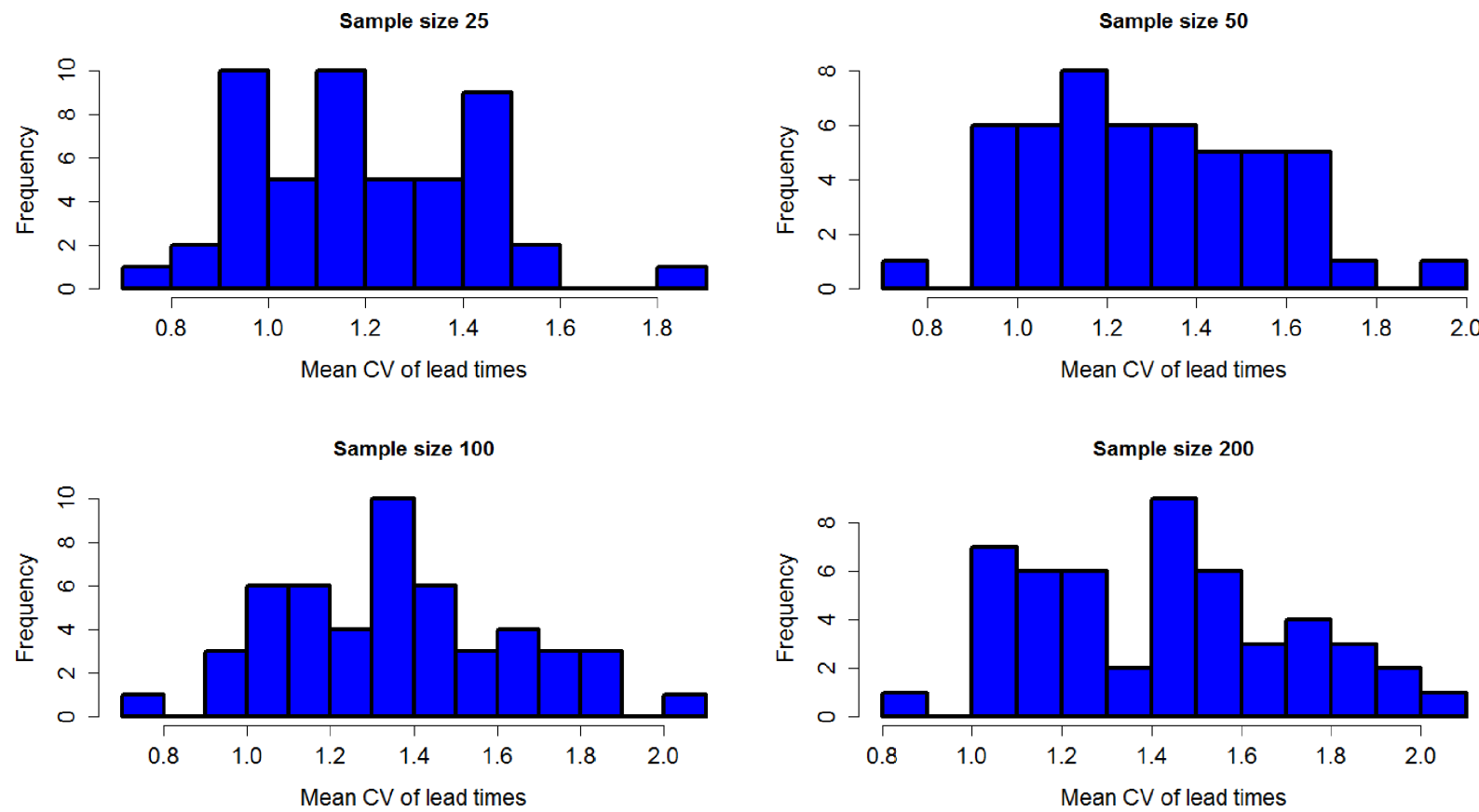

Fig. 2. Median coefficient of variance of lead time distributions for 50 products at various sample sizes. 
Management and Production Engineering Review

In Fig. 3, the 50 investigated products' performance with regards to both independence and whether or not lead time distributions over time can be considered identically distributed is seen. As can be seen from the figure there appears to be a large group accounting for 41 out of the 50 products. These products are found inside the red box. The products' lead times inside the black box can be considered to be strictly i.i.d. with less than 0.2 autocorrelation in mean lead times and more than $80 \%$ of the pairwise comparisons significantly the same. The majority of the products can be found between the black and the red box, where it seems clear that most products have uncorrelated mean lead times, but more samples fail the in the pairwise test. This is interesting, because it underlines that although the lead times do not to a large extend depend on previous observations, the distributions change over time. This has the practical implication that lead time distributions are perhaps best in practice estimated using relative few observations. Figure 3 also illustrates that products that have dependently distributed lead times also have the added complexity that distributions change over time, which of course is linked to the fact that as the means change, so do the distributions. This link also indicates that some products have lead time distributions that are significantly more complicated than i.i.d. However, most interesting is that more than $80 \%$ of the investigated products actually have lead time distributions that conform to the i.i.d. assumption (for a pairwise sample size of 100). Interestingly enough if small sample sizes are considered none of the products have less than $77 \%$ of the comparisons that pass a 0.05 level significance test using Kolmogorov-Smirnov's test, see Table 1.

Dependence and distribution comparison

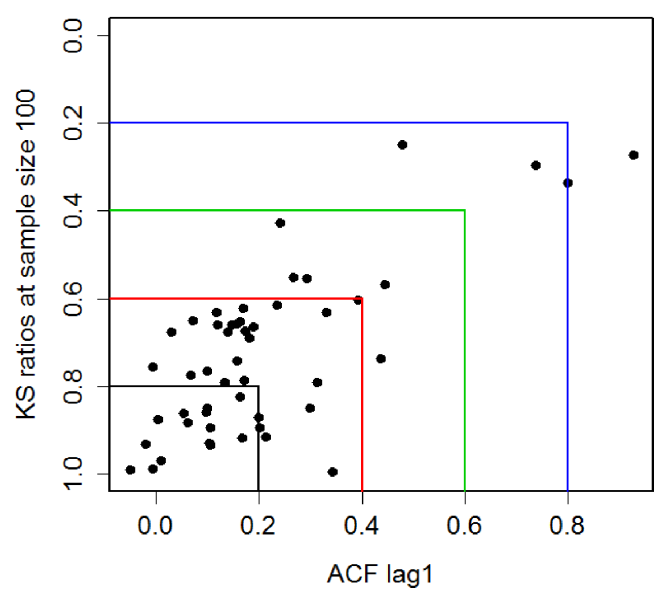

Fig. 3. First order autocorrelation vs. the ratio of samples of size 100 that passes a Kolmogorov-Smirnov (KS) test on a better than 0.05 level.
Table 1

Overview of ratio of samples passing Kolmogorov-Smirnov test for pairwise sample sizes of 25, 50, 100 and 200 for the 50 products.

\begin{tabular}{c|c|c|c|c}
\hline Sample size & 25 & 50 & 100 & 200 \\
\hline Minimum & 0.770 & 0.526 & 0.248 & 0.000 \\
\hline 1st Quartile & 0.840 & 0.746 & 0.637 & 0.429 \\
\hline Median & 0.910 & 0.803 & 0.749 & 0.628 \\
\hline Mean & 0.885 & 0.804 & 0.728 & 0.564 \\
\hline 3rd Quartile & 0.926 & 0.903 & 0.874 & 0.749 \\
\hline Maximum & 0.970 & 0.986 & 0.996 & 1.000 \\
\hline
\end{tabular}

This seems to support the conclusion from the previous section that lead times, when considering short time periods, tend to behave more stable (exhibit less variance) and tend to have the same distributions. This is interesting as it seems to indicate that lead time distributions can be estimated using previous observations, but not too much information as the similarity between lead time distributions decrease significantly when large samples are compared.

Together with the knowledge from the previous investigation of the $\widehat{\sigma}_{t} / \widehat{L}_{t}$ ratio it is clear that in practice lead times must be considered to be stochastic (in this investigation in $100 \%$ of the cases), but luckily it appears that it is enough to assume that they are in fact i.i.d. and do not follow a more complicated structure. This also means that it should be possible to predict lead time distributions for most of the products involved.

\section{Shape of lead time distributions}

Having established that the majority of the lead time distributions can be considered to be i.i.d., the next step is establishing what characterizes a typical lead time distribution in the particular case. From Fig. 4 it is clear that the average lead time distribution is positively (right) skewed. This indicates that the average product's lead time distribution has a long tail of lead time observations. A rule of thumb is that a skewness value above 1 indicates that the distribution is highly skewed. This means that most if not all the investigated products' lead time distributions can be considered to be highly skewed. This corresponds well with the information regarding $\widehat{\sigma}_{t} / \widehat{L}_{t}$ ratios, since large coefficient of variance values tend to be found in skewed distributions. It also indicates a higher likelihood of order crossover occurring (see [21] for a discussion of this phenomenon).

Figure 5 shows the corresponding histograms for kurtosis as Fig. 4 does for skewness. The values are very high, with a marked increase when larger samples are considered. This means that the distribu- 
tions have a large tendency to exhibit peakness and thus have some values of lead times that very frequently occurs. In general, both skewness and kurtosis increase as larger consecutive samples of lead times are considered. This further supports the conclusions drawn in the previous paragraphs, that lead time distributions are more stable if shorter time pe- riods are considered. It seems reasonable to conclude that if relative few observations (25-50) are used, the majority of the products' lead time distributions can be adequately predicted for the subsequent period. This means that it should be possible to use these distributions for planning purposes, given that they are continuously updated.
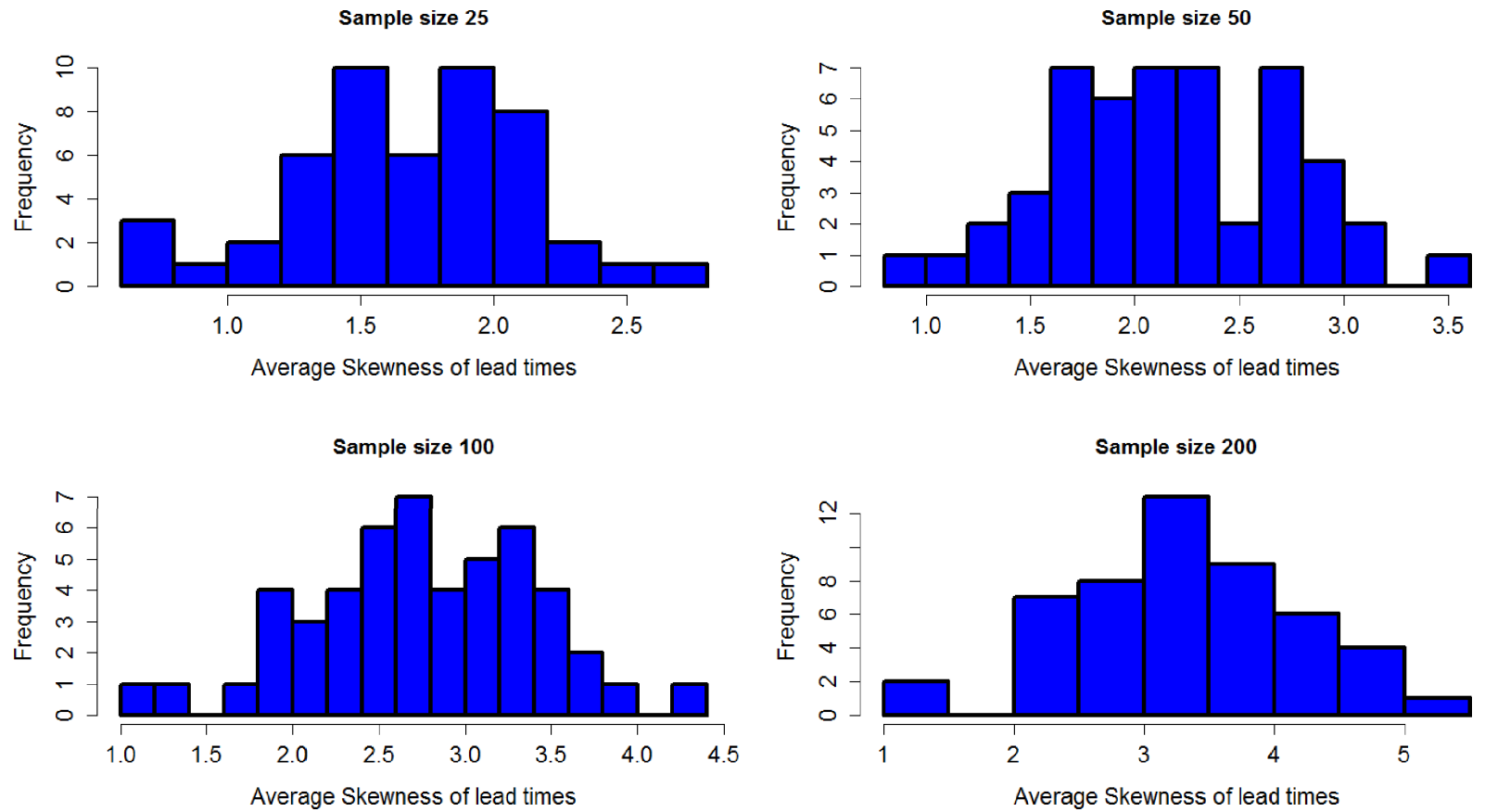

Fig. 4. Histogram of median skewness for 50 products in consecutive samples of size; 25, 50, 100 and 200.
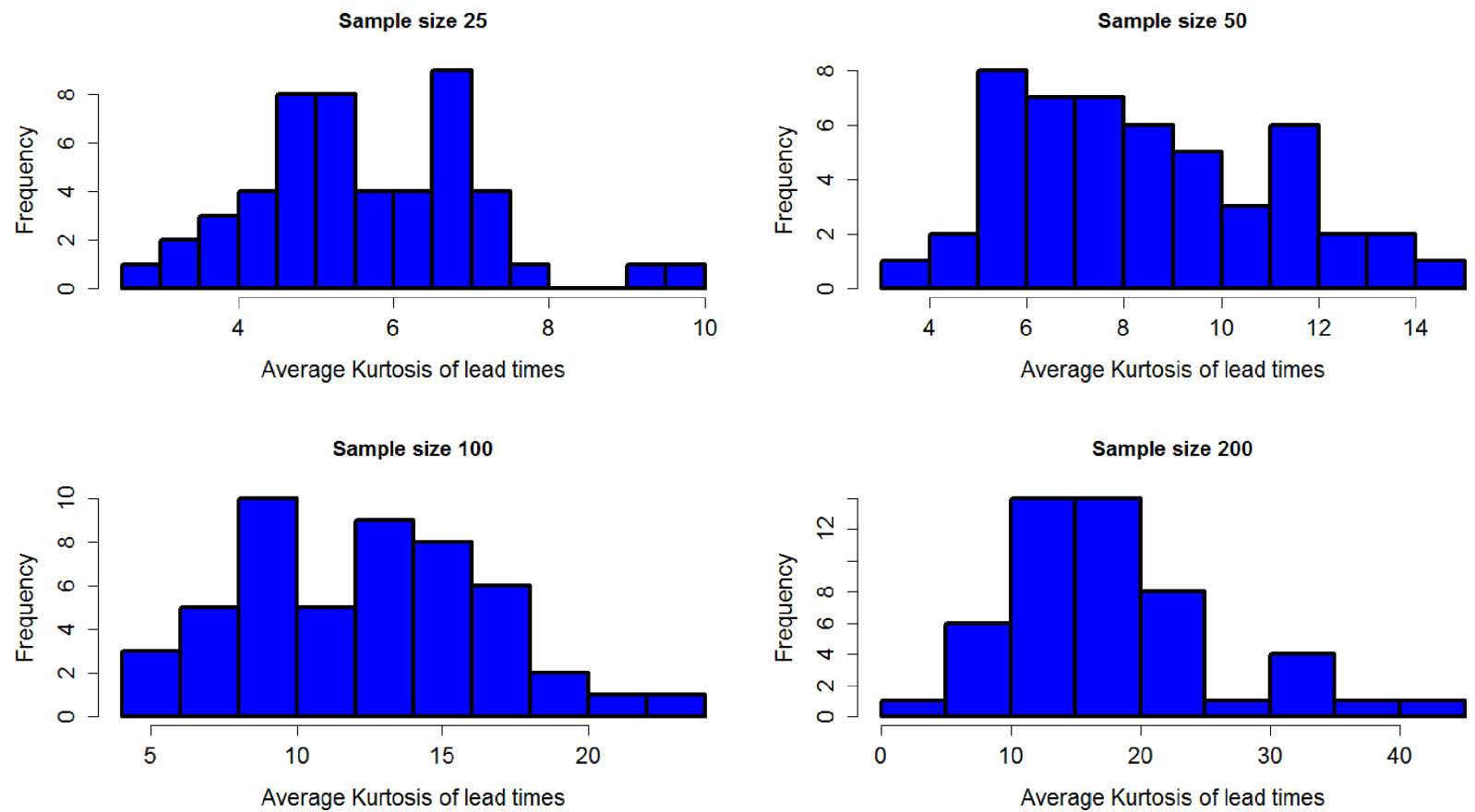

Fig. 5. Histogram of median kurtosis skewness for 50 products in consecutive samples of size; 25, 50, 100 and 200. 
To illustrate a typical lead time distribution from the investigated case an example density function with the following parameters skewness 3 , kurtosis 17 and $\widehat{\sigma}_{t} / \widehat{L}_{t} 0.95$ is shown in Fig. 6. The shape of the lead time distribution shown in Fig. 6 has the typical parameters found in the investigated data.

\section{Concept plot of lead time distribution}

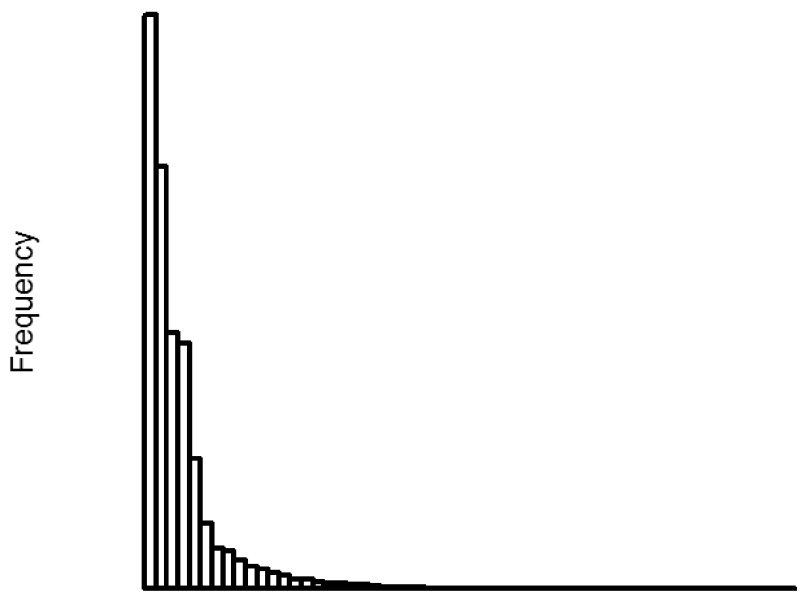

Lead time

Fig. 6. Example density function for a typical lead time distribution with the average characteristics found through the study.

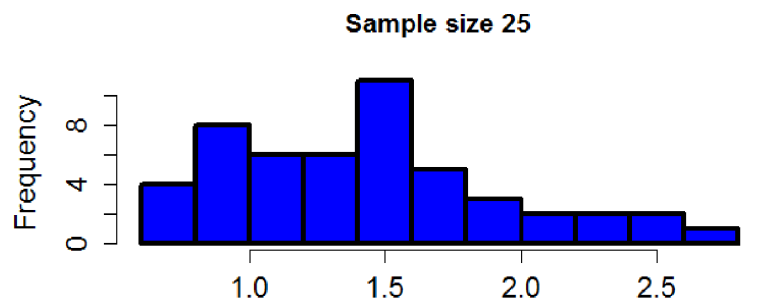

Average increase in variance of lead times demand

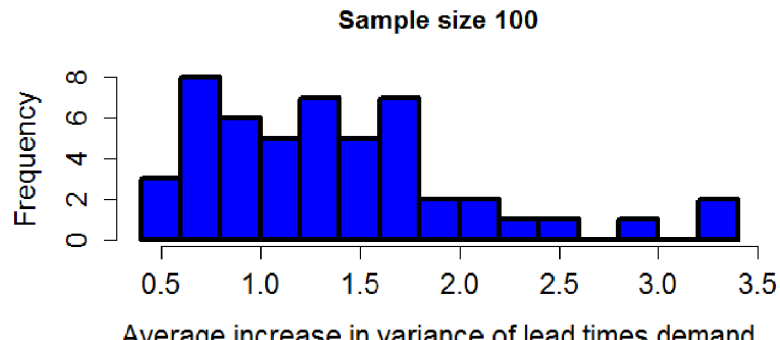

Fig. 7. Average increase in variance of lead time demand due to lead time variance.

\section{Lead time demand variance from stochastic lead times}

Having established that the lead time distributions are very volatile, but still for the most part conform to the assumption of i.i.d. behavior, it now becomes possible to estimate how much lead time demand variance is influenced by the stochastics of lead times. The graphs in Fig. 7 illustrate that in the investigated case on average more lead time demand variance is incurred due to variance in lead times, than due to variance in demand.

In general, the company should expect to experience more than double the lead time demand variance when stochastic lead times are considered. This is interesting for several reasons. First it may explain if the company experiences larger variance in service level than expected. Second, it underlines that neglecting the lead time variance leads to an underestimation of the actual lead time demand variance, and thus to holding significantly less safety stock than required. Third, it strongly indicates that the company could achieve a performance improvement by reducing lead time variance, potentially more so than by reducing demand variance or average lead times. The findings support theory in the sense that Chaharsooghi and Heydari [10] find that reducing lead time variance is more important in certain settings than reducing mean lead time.

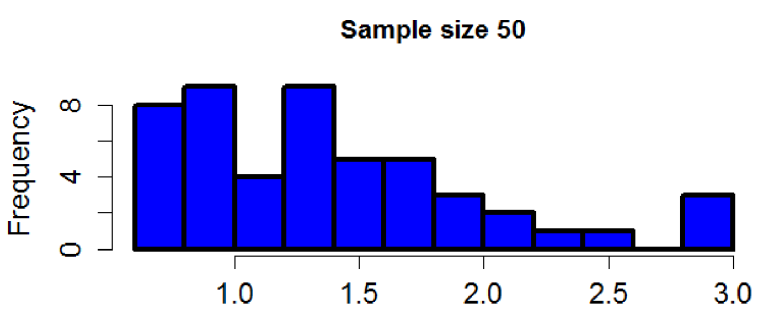

Average increase in variance of lead times demand

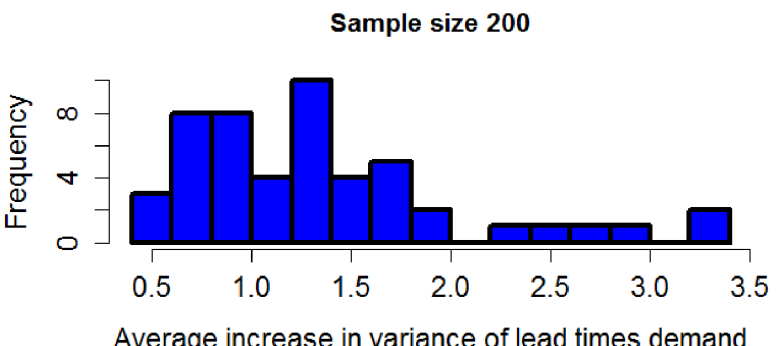


Table 2

Overview of $\Delta S S$ for sample sizes of $25,50,100$ and 200 for the 50 products.

\begin{tabular}{c|c|c|c|c}
\hline Sample size & 25 & 50 & 100 & 200 \\
\hline Minimum & 0.74 & 0.64 & 0.50 & 0.46 \\
\hline 1st Quantile & 1.05 & 0.89 & 0.84 & 0.85 \\
\hline Median & 1.42 & 1.27 & 1.27 & 1.25 \\
\hline Mean & 1.43 & 1.37 & 1.36 & 1.34 \\
\hline 3rd Quantile & 1.72 & 1.73 & 1.69 & 1.67 \\
\hline Maximum & 2.74 & 2.96 & 3.30 & 3.33 \\
\hline
\end{tabular}

In all the investigations the total amount of observations available and their potential influence on the results have not been discussed. Figure 8 illustrates scaled demand and lead time uncertainty for the 50 products. Different colors are used for the ten most frequently sold products, the 11-20 most sold and so on. There seems to be no clear link between the order frequency and the variance experienced, which is supported by correlation analysis that shows a non-significant correlation between the number of observations and the scaled uncertainty. However, it is interesting to note that there is a strong correlation (0.9) between the uncertainty generated by demand variance and by lead time variance. To underline this, the correlations between the number of available observations and; skewness, kurtosis, $\widehat{\sigma}_{t} / \widehat{L}_{t}$ ratio and dependence between observations, with no significant correlations found. This means that the number of observations available (or to be more precise the frequency with which a product is sold) has no influence on the product's lead time behavior. This fact is interesting as one could expect that there would be some form of dependence. However, this is also seems to be an indirect support of the fact that most of the investigated lead time distributions truly seem to be i.i.d.

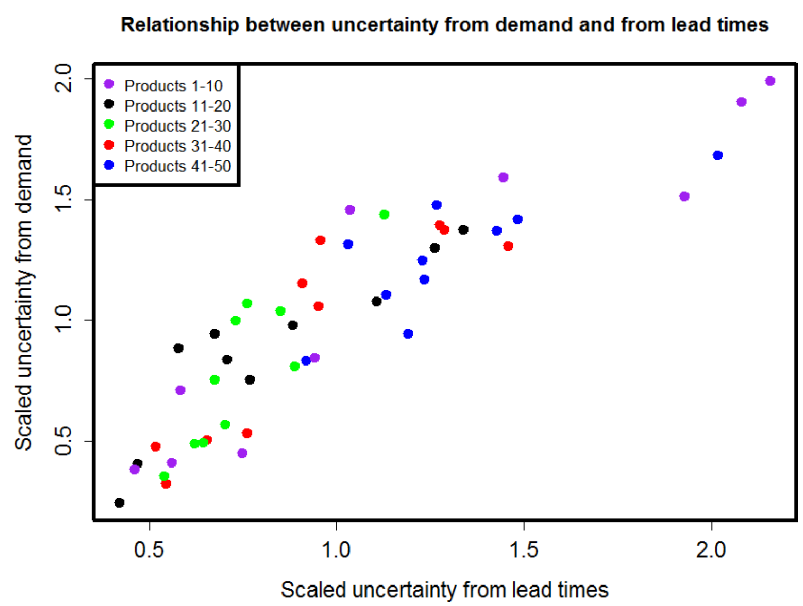

Fig. 8. Scaled uncertainty of demand and lead time with indication of product order frequency.

\section{Managerial challenges}

The main practical insight from this analysis of lead times is that it is critical to address the lead time uncertainty in the planning and control approaches in the company. Especially as it appears quite obvious in this particular case lead time demand variance depends more on lead time variance than on demand variance.

The obvious conclusion in practice must then be that company must compensate in their planning \& control for this added uncertainty. This can be managed through using optimization techniques [25, 26 ] or through re-design of the chain (e.g. stabilizing the system through achieving periodic cycles of stable behavior [27]). The challenge is of course how to go about this without dramatically increasing safety stock and thus inventory holding cost and implicitly more variance and thus bullwhip effect to the supply chain. It also seems to support the conclusions found in [10], who conclude that to reduce bullwhip effect in supply chains experiencing stochastic lead times, one should reduce the variance of lead times. This conclusion is mirrored in the work of Michna et al. [16], who conclude that stochastic lead times and the subsequent need to forecast expected lead times at any given tier in the supply chain is a major source of bullwhip effect.

It also raises a number of practical considerations internally in the company. First, how is the uncertainty from lead times affecting the daily operations in form of increasing stock or stock outs? The planning organizations have options to include stochastic characteristics - but in practice Stochastic lead times are only sporadically taken into account in the company's inventory management, the practical question then becomes if this uncertainty is implicitly if not explicitly included in the setting of Re-order-Points and order quantities - and how. In practice it is neigh on impossible to determine how employees perceive the lead time uncertainty as currently the knowledge in the organization is tacit not explicit. However, just by focusing on the area the uncertainty goes from being intuitively true, to explicit and quantifiable. This is issue is further compounded by the inherent need to aggregate demand information [28]. Second, it raises the important question of how to address lead times. If they are stochastic, as is in the case, theory would suggest removing variance is the best way to reduce bullwhip effect. If nothing else the investigation of the lead times have confirmed the company in that their ongoing focus on lead-time targets and stabilization - thus reducing lead time variance is in fact the correct way to go - and good results 
are starting to appear. In the context of a multi-level supply chain the historical data is impacted by managerial stock and capacity decisions leading to leadtime fluctuations from one steady state to another - thus introducing variance in the data. It has also raised interesting questions of how to improve the work with step changes in a deep multi-level supply chain environment, how to monitor lead times and what is needed with regards to data discipline and competence to support this. So the analysis of lead times has actually supported ongoing initiatives in the company, in the sense of confirming the importance of reducing variability in lead times. Here the skewness of the lead time distributions has been of large importance, as skewed lead time distributions tend to lead to more difficult (unstable) planning environments. In general, the study has led to an increased focus on lead times in the company. Moving forward the emphasis is on determining first the impact on the company's supply chain and second to adapt the supply chain setup accordingly. Further steps could also include using e.g. RFID technology to improve visibility and data quality throughout the supply chain [29].

\section{Conclusions}

Summarizing the findings for the lead time distributions in the investigated case, the following general characteristic can be given. Lead times are stochastic and for the large majority of products behave as i.i.d. variables. The distributions tend to be highly skewed, and uniformly right skewed i.e. having a long tail of observations. Likewise, the lead time distributions in all cases exhibit a large degree of peakness (kurtosis), indicating that some lead times occur with a very high frequency. Taken together the lead time distributions of the particular case seem to be highly volatile, making for a tricky planning environment. This is confirmed by the fact that on average lead times contribute $35 \%$ more than demand to lead time demand variance. This also highlights the fact that in practice it may well be worth reducing lead time variance rather than the mean lead time. It also appears that lead time distributions can with a relative large degree of certainty be estimated the newest information available. This has the practical implication that companies should monitor their lead times (both from suppliers and to customers) and use the updated lead time information in their planning.

The question is to which extent the observations from this particular case can be used to draw general conclusions of lead time behavior in other supply chains? Certainly there is no foundation to claim that all lead time distributions experienced in companies behave in a similar manner. However, the results within this company where very consistent across 50 products, with similar conclusions being reached for the vast majority of the products. At the very least the example here can serve to illustrate how faulty the assumption of deterministic lead times is in practice, and the price in underestimation of lead time demand variance paid through this assumption. There is no doubt that lead times in most real life settings are in fact stochastic and that assuming that they are constant can at best lead to suboptimal performance of the supply chain or at worst lead to implementing the wrong improvement measures. However, there is also little doubt that the particular structure of lead time distributions will depend on the context, as will the impact of the stochastic behavior on the particular supply chain. In this sense the presented research can be considered as a method for determining the lead time behavior in a given case and thus enable companies to analyze their own lead times both up and down stream.

Future research will focus on establishing whether there are other factors that significantly influence the lead time distributions, with a special emphasis on whether or not the lead times to some extend depend on order sizes.

This work has been partly supported by the National Science Centre grant 2012/07/B//HS4/00702.

\section{References}

[1] Otto A., Kotzab H., Does supply chain management really pay? Six perspectives to measure the performance of managing a supply chain, European Journal of Operational Research, 144, 2, 306-320, 2003.

[2] Singh R.K., Sharma M.K., Prioritising the alternatives for flexibility in supply chains, Production Planning \& Control, 25, 2, 176-192, 2014.

[3] Chen F., Drezner Z., Ryan J.K., Simchi-Levi D., Quantifying the bullwhip effect in a simple supply chain: The impact of forecasting, lead times, and information, Management Science, 46, 3, 436-443, 2000.

[4] Mascle C., Gosse J., Inventory management maximization based on sales forecast: case study, Production Planning \& Control, 25, 12, 1039-1057, 2014.

[5] Silver E.A., Pyke D.F., Peterson R., Inventory management and production planning and scheduling, Wiley New York, Vol. 3, 1998.

[6] Nielsen P., Michna Z., Do N.A.D., An empirical investigation of lead time distributions, IFIP Interna- 
tional Conference on Advances in Production Management Systems, Springer 2014.

[7] Chatfield D.C., Kim J.G., Harrison T.P., Hayya J.C., The bullwhip effect - impact of stochastic lead time, information quality, and information sharing: a simulation study, Production and Operations Management, 13, 4, 340-353, 2004.

[8] Kim J.G., Chatfield D., Harrison T.P., Hayya J.C., Quantifying the bullwhip effect in a supply chain with stochastic lead time, European Journal of Operational Research, 173, 2, 617-636, 2006.

[9] Bagchi U., Hayya J.C., Chu C.-H., The effect of lead-time variability: the case of independent demand, Journal of Operations Management, 6, 2, 159-177, 1986.

[10] Chaharsooghi S.K., Heydari J., LT variance or LT mean reduction in supply chain management: which one has a higher impact on SC performance?, International Journal of Production Economics, 124, 2, 475-481, 2010.

[11] Wikner J., Towill D.R., Naim M., Smoothing supply chain dynamics, International Journal of Production Economics, 22, 3, 231-248, 1991.

[12] Metters R., Quantifying the bullwhip effect in supply chains, Journal of Operations Management, 15, 2, 89-100, 1997.

[13] Lee H.L., Padmanabhan V., Whang S., The bullwhip effect in supply chains, MIT Sloan Management Review, 38, 3, 93, 1997.

[14] Lee H.L., Padmanabhan V., Whang S., Information distortion in a supply chain: the bullwhip effect, Management Science, 43, 4, 546-558, 1997.

[15] McCullen P., Towill D., Diagnosis and reduction of bullwhip in supply chains, Supply Chain Management: An International Journal, 7, 3, 164-179, 2002.

[16] Michna Z., Nielsen I.E., Nielsen P., The bullwhip effect in supply chains with stochastic lead times, Mathematical Economics, 9, 16, 71-88, 2013.

[17] Song J.-S., The effect of leadtime uncertainty in a simple stochastic inventory model, Management Science, 40, 5, 603-613, 1994.

[18] Buchmeister B., Pavlinjek J., Palcic I., Polajnar A., Bullwhip effect problem in supply chains, Advances in Production Engineering \& Management, 3, 1, 45$55,2008$.
[19] Ghaffari M., Javadian N., Analysis of the bullwhip effect with multi-echelon, multi-product supply chains, International Journal of Industrial Engineering Computations, 4, 4, 579-586, 2013.

[20] Wang X., Disney S.M., Mitigating variance amplification under stochastic lead-time: the proportional control approach, European Journal of Operational Research, 256, 1, 151-162, 2017.

[21] Disney S.M., Maltz A., Wang X., Warburton R.D.H., Inventory management for stochastic lead times with order crossovers, European Journal of Operational Research, 248, 2, 473-486, 2016.

[22] Gross D., Soriano A., The effect of reducing leadtime on inventory levels-simulation analysis, Management Science, 16, 2, B-61-B-76, 1969.

[23] Eppen G.D., Martin R.K., Determining safety stock in the presence of stochastic lead time and demand, Management Science, 34, 11, 1380-1390, 1988.

[24] So K.C., Zheng X., Impact of supplier's lead time and forecast demand updating on retailer's order quantity variability in a two-level supply chain, International Journal of Production Economics, 86, 2, 169-179, 2003.

[25] Sitek P., Wikarek J., A hybrid framework for the modelling and optimisation of decision problems in sustainable supply chain management, International Journal of Production Research, 53, 21, 6611-6628, 2015 .

[26] Sitek P., Nielsen I.E., Wikarek J., A hybrid multiagent approach to the solving supply chain problems, Procedia Computer Science, 35, C, 1557-1566, 2014.

[27] Bocewicz G., Banaszak Z.A., Declarative approach to cyclic steady state space refinement: periodic process scheduling, The International Journal of Advanced Manufacturing Technology, 67, 1-4, 137$155,2013$.

[28] Eriksen P.S., Nielsen P., Order quantity distributions: estimating an adequate aggregation horizon, Management and Production Engineering Review, 7, 3, 39-48, 2016.

[29] Nielsen I., Lim M., Nielsen P., Optimizing supply chain waste management through the use of RFID technology, Proceedings of 2010 IEEE International Conference on RFID-Technology and Applications, RFID-TA, pp. 296-301, 2010. 